\title{
Análisis de la colaboración entre las empresas biotecnológicas españolas con actividades de I+D y el sistema público de I+D
}

\author{
Esther García-Carpintero*, Armando Albert-Martínez*, Begoña Granadino*, Luis M. Plaza* \\ *Instituto de Estudios Documentales sobre Ciencia y Tecnología (IEDCYT) - Consejo Superior de Investigaciones Científicas (CSIC). \\ Correo-e: esther.carpintero@cchs.csic.es
}

Recibido: 01-07-2013; 2a version: 10-09-2013; Aceptado: 21-09-2013.

Cómo citar este artículo/Citation: García-Carpintero, E.; Albert-Martínez, A.; Granadino, B.; Plaza, L.M. (2014). Análisis de la colaboración entre las empresas biotecnológicas españolas con actividades de I+D y el sistema público de I+D. Revista Española de Documentación Científica, 37(2):e041. doi: http://dx.doi.org/10.3989/redc.2014.2.1106

Resumen: El desarrollo y la mejora de la cooperación entre el sector productivo y el sistema público de I+D puede generar un conjunto de ventajas competitivas para estas empresas. El presente trabajo describe las experiencias y percepciones de las empresas biotecnológicas españolas en su colaboración con el sistema público de I+D. Los resultados muestran que prácticamente la totalidad de empresas biotecnológicas españolas con actividades de I+D (un 93,6\%) han mantenido algún tipo de colaboración con el sistema público de I+D. Los principales obstáculos percibidos por estas empresas en su colaboración con el sistema público de I+D son el exceso de burocracia y la respuesta lenta de los investigadores. Los resultados obtenidos sugieren la conveniencia de establecer políticas y programas públicos para fomentar la transferencia de conocimiento y tecnología desde el sistema público de I+D a las empresas, fundamentalmente centrados en la necesidad de incentivar a los investigadores del sistema público de I+D y mejorar el funcionamiento de las Oficinas de Transferencia de Tecnología.

Palabras clave: Biotecnología; transferencia de tecnología; innovación; sistema público de I+D; cooperación industria-academia.

Analysis of the collaboration between Spanish biotechnological companies with R\&D activities and the public R\&D system

\begin{abstract}
The development and improvement of cooperation between the industrial sector and the public R\&D system can produce competitive advantages for the companies. The present work describes experiences and perceptions of Spanish biotechnology companies in their collaboration with the public R\&D system. Results show that almost all the biotechnological companies with R\&D activities (93.6\%) have cooperated with the public R\&D system. The main barriers found by the companies are the excess of bureaucracy and the slow response of public researchers. Results obtained also suggest the need to establish public policies and programmes to promote knowledge and technology transfer from public research centres to companies, with a special focus on encouraging researchers from the public R\&D system and on improving how Technology Transfer Offices work.
\end{abstract}

Keywords: Biotechnology; technology transfer; innovation; public R\&D; industrial-academic cooperation.

Copyright: (C) 2014 CSIC. Este es un artículo de acceso abierto distribuido bajo los términos de la licencia Creative Commons Attribution-Non Commercial (by-nc) Spain 3.0. 


\section{INTRODUCCIÓN}

La mejora de los procesos productivos y la competitividad de las industrias es una de las prioridades de las políticas nacionales de muchos países. La cooperación entre la industria y el sistema público de I+D es uno de los mecanismos principales para facilitar la innovación tecnológica y la competitividad de las empresas. Una mayor colaboración empresa-academia permite mejorar la capacidad innovadora del sector productivo, la creación de nuevas empresas, la formación e incorporación de personal altamente cualificado a las empresas, etc. (Etzkowitz y Leydesdorff, 2000).

El desarrollo y mejora de vínculos entre el sector productivo y el sistema público de I+D puede generar una serie de ventajas generales, tanto a la empresa como a las universidades o centros implicados. Para la empresa, las ventajas esperables son, entre otras, el aumento del número de patentes, la resolución de problemas en la investigación o en la producción, el acceso a nuevos conocimientos y redes de investigación, el aumento de la productividad, facilitar el acceso a fondos de investigación, y el acceso a profesionales especializados (Schartinger y otros, 2002; Santoro y Gopalakrishnan, 2000). Otros estudios muestran que las empresas con un alto grado de colaboración con las universidades y organismos públicos de investigación (OPIs) son más competitivas (Hayton y otros, 2010).

Por otro lado, la comunidad científica puede obtener de esta colaboración una mayor calidad de la investigación o de la enseñanza universitaria, nuevos desarrollos científicos y sus aplicaciones, ampliar el acceso a los fondos para la I+D, etc. (Ramos Vielba y otros, 2009). Esta colaboración también puede facilitar la incorporación posterior de licenciados o ingenieros a las empresas, permite ofrecer a los estudiantes universitarios realizar prácticas en empresas, la obtención de becas de formación, etc. (Yong, 2000).

Existen numerosos ejemplos de la capacidad del sistema público de I+D en el desarrollo de innovaciones mediante la obtención de patentes o la creación de empresas de base tecnológica, creando un valor de mercado importante y empleos de alta cualificación (George y otros, 2002). En el caso de las industrias basadas en conocimiento científico como la industria biotecnológica, donde la innovación continua es una prioridad estratégica, las alianzas con el sistema público de I+D son muy importantes para el desarrollo de la actividad empresarial (Albert y Plaza, 2004; Al-Laham y otros, 2011). Esta colaboración con el sistema público permite a las compañías biotecnológicas adquirir el conocimiento que contribuye a aumentar de forma importante su nivel de competitividad e innovación (Oliver, 2004; Zaheer y George, 2004; Vega-Jurado y otros, 2009; Almeida y otros, 2011).
A pesar de las ventajas mutuas que genera la cooperación entre el sistema público de I+D y la industria, la cooperación no está exenta de dificultades y el establecimiento de vínculos estables y duraderos es difícil (Albert y otros, 2007). Uno de los problemas más importantes que pueden surgir es la distinta cultura en la difusión de conocimiento que se produce entre ambos actores: frente a la libre difusión del conocimiento de universidades y centros públicos de investigación, estaría el secreto o protección industrial de las empresas (George y otros, 2002). Otros impedimentos están relacionados con el exceso de burocracia, los problemas en el cumplimiento de los plazos, etc.

Las oficinas de transferencia de tecnología (OTTs) del sistema público de I+D, implantadas en universidades y organismos públicos de investigación (OPIs), se crearon para desempeñar un papel clave en este tipo de colaboración. Estas OTTs actúan de intermediarias entre las dos partes, facilitan la formalización de contratos entre las empresas y los investigadores y realizan una labor de difusión de los resultados de investigación de sus instituciones a las empresas.

Para fomentar y mejorar la cooperación entre el sistema productivo y el sistema público de $I+D$, los gobiernos y agencias de investigación involucradas en la toma de decisiones necesitan disponer de una serie de indicadores que les permita conocer cuáles son los determinantes principales en dichas colaboraciones, cómo se establecen, en qué áreas se producen de forma predominante, y cuáles son las barreras u obstáculos más importantes que se han encontrado en esa colaboración.

La mayoría de los estudios publicados sobre colaboración entre el sector productivo y el sector público de I+D están centrados en las motivaciones de los investigadores y se basan en el análisis de la co-autoría de publicaciones científicas o de patentes (Zhang y otros, 2007; Abramo y otros, 2009; Thursby y Thursby, 2011). Las actividades de colaboración, medida en términos de co-autoría, se basan en la suposición de que las empresas publican lo suficiente como para proporcionar información estadística representativa sobre las características de su colaboración. Sin embargo, en muchos casos la colaboración entre las empresas y el sector público de I+D no da lugar a publicaciones científicas o patentes, sobre todo en los casos en que las empresas contratan a universidades u OPIs para solucionar problemas puntuales o cuando así lo requiera el secreto industrial. Por tanto, este tipo de metodología no es adecuado para la medición de todos los tipos de cooperación que se pueden establecer entre el sector público y la industria (Katz y Martin, 1997). Otros estudios, por su parte, se han centrado en el papel de las OTTs (Martinelli y otros, 2008; Muscio, 2010; Cardozo y otros, 2011). Sin embargo, en muy pocas ocasiones, se ha analizado la cooperación del sistema público de I+D con las empresas, desde el punto de vista de estas últimas. 
El presente estudio pretende analizar en profundidad la cooperación del sector biotecnológico español con el sistema público de I+D a partir del análisis de una encuesta distribuida a las empresas. Este trabajo pretende definir las características principales del sector biotecnológico español con actividades en I+D (tipo de actividad, número de empleados, si ha recibido subvenciones públicas para la I+D, etc.) y establecer cuál es la situación actual de la cooperación entre el sector biotecnológico y el sistema público de I+D y, a partir de ahí, cómo podría seguir fomentándose esta colaboración. Se analizará cuál es el tipo de cooperación más habitual, el grado de satisfacción con las cooperaciones anteriores, las principales barreras encontradas, la intención de establecer nuevas colaboraciones en un futuro inmediato, etc.

\section{METODOLOGÍA}

La colaboración entre las empresas biotecnológicas españolas con el sistema público de $I+D$ se ha analizado mediante el diseño, distribución y análisis de una encuesta a las empresas biotecnológicas españolas con actividades en I+D. El cuestionario está dividido en dos secciones. La primera se centra en determinar las características de las empresas biotecnológicas que realizan actividades I+D en términos de tamaño, uso de biotecnologías, sectores biotecnológicos en los que opera, si es beneficiaria de ayudas públicas, etc. La segunda parte del cuestionario se centra en las actividades de colaboración con el sistema público de I+D como el tipo de colaboración, el grado de satisfacción con la misma y los obstáculos encontrados en esa colaboración. En el diseño de la encuesta se han tenido en cuenta las recomendaciones de definición estadística de la biotecnología propuestas por el documento de la OCDE "A Framework for Biotechnology Statistics" (OCDE, 2005a).

La distribución de este cuestionario se hizo mediante correo electrónico de Junio a Septiembre de 2009, junto con una carta de solicitud de colaboración y presentación del objetivo académico y el carácter puramente estadístico de la misma. Un aspecto importante en este estudio fue la selección de las empresas biotecnológicas con actividades en $I+D$ a las que se distribuiría esta encuesta. De forma genérica es muy difícil determinar el número total de empresas con actividades en el sector biotecnológico y particularmente el número de empresas con actividades en I+D, dada la dificultad en definir de forma estadística las actividades biotecnológicas.

En el caso del número total de empresas biotecnológicas españolas, los informes disponibles en la bibliografía ofrecen cifras muy diferentes. Por ejemplo, atendiendo a la definición de empresa biotecnológica propuesta por la OCDE "aqueIla cuya actividad principal incluye la aplicación de técnicas biotecnológicas para producir bienes, servicios y/o desarrollo de I+D" (OCDE, 2005b), la OCDE estimó que en 2010 había 617 empresas biotecnológicas españolas en su informe "OECD Key Biotechnology Indicators 2010" (OCDE, 2012), mientras que la Fundación Genoma España fija este número en 395 (Fundación Genoma España, 2011); por otra parte, la Asociación Española de Bioempresas tenía un total de 250 socios en 2012 (ASEBIO, 2013). Sin embargo, no todas las empresas biotecnológicas tienen actividades en I+D, y son precisamente estas últimas las que son de interés para este trabajo, puesto que se pretende evaluar cómo interactúan con el sistema público de I+D. En el informe ASEBIO 2011 (ASEBIO 2012) se afirmaba que en total había sólo 54 empresas biotecnológicas que realizaban $\mathrm{I}+\mathrm{D}$, sin embargo la Fundación Genoma España estimaba ya en 146 el número de empresas que realizan actividades de I+D sólo en el sector de la salud.

Para este estudio los autores ha desarrollado una base de datos con las empresas biotecnológicas españolas que realizan actividades en I+D a partir de los listados de socios de Asociación Española de Bioempresas (ASEBIO) y de la Fundación Genoma España. En total se han identificado 250 empresas con actividades en I+D de un total de 441 empresas españolas parcial o totalmente dedicadas a la biotecnología recogidas en ambos listados.

El número total de respuestas recibidas de empresas biotecnológicas con actividades en I+D fueron 78 (un $31,2 \%$ del total de empresas a las que se le envió el cuestionario). El error muestral calculado, al $95 \%$ de grado de confianza, es del $8,55 \%$. El número de empresas que han contestado la encuesta es, por una parte, superior al número de 54 empresas biotecnológicas que realizaban I+D de acuerdo al Informe ASEBIO 2010 (ASEBIO 2011) y, por otra parte, significaría un $53,4 \%$ de las empresas que realizan actividades de I+D de acuerdo al Informe Genoma. Por tanto, a pesar del error muestral calculado, suponiendo un universo de 250 empresas, este número de respuestas puede considerarse como muy importante, especialmente porque lo primero que se les preguntaba en el cuestionario era si tenían o no actividades en I+D y si no era el caso, se les pedía no seguir con la encuesta. Tampoco se ha observado un sesgo muestral ni por tamaño de empresa, ni por importancia de sus actividades biotecnológicas o áreas de actividad, de acuerdo a los informes disponibles sobre el sector biotecnológico español de ASEBIO y de la Fundación Genoma España, tal y como se comentará al inicio de la sección de resultados y discusión.

El análisis de las encuestas se ha llevado a cabo mediante el software estadístico SPSS 19 (IBM). Se ha analizado tanto el porcentaje de respuestas con respecto al número total de respuestas en las diferentes preguntas del cuestionario como diferentes estadísticos, que permiten analizar tanto la dependencia o interdependencia entre variables como la magnitud de esta asociación o correlación. En con- 
creto, se ha utilizado la prueba Chi-cuadrado para determinar si dos variables cualitativas son o no independientes con un determinado nivel de confianza. Por otra parte, se ha utilizado el coeficiente de correlación de Spearman o Spearman-rho para analizar la magnitud y sentido de la asociación entre variables. Este coeficiente varía entre -1 y 1 , indicando asociaciones negativas o positivas, respectivamente; valores próximos a cero indicarán que no hay asociación lineal, lo que no significa que no pueda existir otro tipo de asociación. Al igual que el thau-b de Kendall, el coeficiente de correlación de Spearman puede utilizarse como una alternativa al de Pearson cuando las variables estudiadas son ordinales y/o se incumple el supuesto de normalidad.

\section{RESULTADOS Y DISCUSIÓN}

3.1 Descripción de las empresas biotecnológicas españolas con actividades de I+D

Tamaño de las empresas e importancia de las actividades biotecnológicas. En primer lugar, se preguntó a las empresas por su número de empleados y por el número de empleados dedicados a actividades relacionadas con la biotecnología. En este sentido, se constató que un $67,9 \%$ de las empresas biotecnológicas que realizan actividades de I+D tienen menos de 25 empleados. Un importante número de empresas $(48,7 \%)$ pueden considerarse micro-empresas, al tener menos de 10 empleados. Las 78 empresas que contestaron a la encuesta suman un total de 1.800 empleados y declaran que el $71 \%$ de este personal está dedicado exclusivamente a actividades biotecnológicas. Estos datos concuerdan tanto con los datos ofrecidos por la Asociación Española de Bioempresas - ASEBIO (ASEBIO, 2011) como por la Fundación Genoma España (Fundación Genoma España, 2011), que indican que la mayoría de compañías biotecnoló- gicas son de pequeño tamaño (con un promedio de 16 trabajadores) y que aproximadamente tres cuartas partes del personal de estas empresas están dedicados a actividades biotecnológicas.

La importancia estratégica de las actividades biotecnológicas en las empresas fue considerada como "crucial o muy importante" por el $78 \%$ de las compañías, lo que indica que la mayoría de las empresas tienen a la biotecnología como actividad principal o son empresas completamente dedicadas a la biotecnología, como a veces también se las denomina. El $17 \%$ de las empresas consideraban la biotecnología como "importante" en sus actividades y sólo un $5 \%$ consideró que su biotecnología tenía una importancia menor en sus actividades. Tras ser preguntadas por el efecto que habían tenido sus actividades biotecnológicas en los beneficios o pérdidas de los últimos años, un $72 \%$ de las mismas consideraron que la actividad biotecnológica había producido beneficios, mientras que en un $28 \%$ había producido pérdidas.

Tipos de biotecnologías utilizadas por las empresas. Con respecto a los tipos de biotecnologías empleadas por las empresas, la figura 1 muestra el porcentaje de empresas que emplearon cada una de las biotecnologías tal y como se clasifican en la definición-lista de la OCDE (OCDE, 2005b). Las biotecnologías más empleadas por las empresas son, en primer lugar, las relacionadas con el DNA/RNA (64,1\% de las empresas), y después, las relacionadas con proteínas y macromoléculas ( $48,7 \%$ de las empresas), los procesos biotecnológicos $(43,6 \%)$ y el cultivo e ingeniería de células y tejidos $(41,0 \%)$. Por otra parte, la bioinformática y la nanobiotecnología son empleadas por un $30,8 \%$ y un $26,9 \%$, respectivamente. Finalmente, ya de menor importancia, son el empleo de genes o vectores RNA $(10,3 \%)$ y otras biotecnologías $(14,1 \%)$.

Figura 1. Biotecnologías usadas por las empresas españolas de acuerdo a la definición-lista de la OCDE

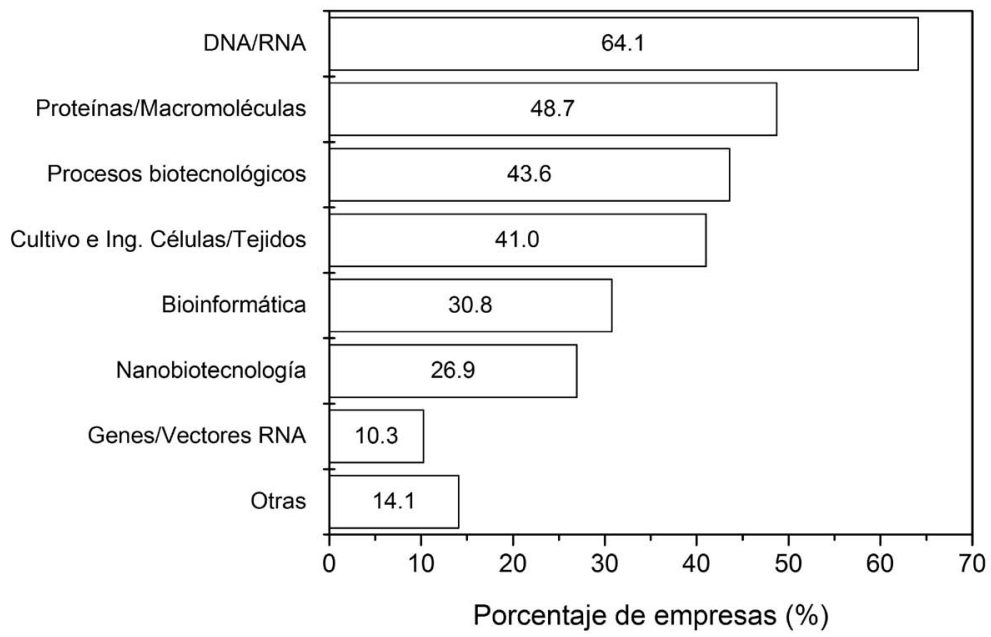


Áreas de actividad biotecnológica en actividades de $I+D$, producción/comercialización de bienes y servicios y en el uso como "input". Para conocer más profundamente las áreas de la biotecnología involucradas en los diferentes tipos de actividad de las empresas, se les pidió que identificaran las áreas de la biotecnología en las que estaban involucradas sus compañías, distinguiendo si esas áreas eran importantes en sus actividades de $I+D$, en actividades de producción/comercialización de bienes o servicios, o si eran utilizadas como "input" para su actividad. Las áreas de la biotecnología analizadas fueron: la salud humana, los procesos industriales, la agricultura, la bioinformática, el medio ambiente, aplicaciones no específicas y otros. En la figura 2 se representa el porcentaje de empresas con actividades en cada una de estas áreas, referido al total de empresas encuestadas.

En primer lugar es necesario comentar que todas las empresas seleccionadas para esta encuesta tienen actividades en $I+D$, mientras que sólo un $74,0 \%$ de las empresas tienen actividades de producción/comercialización y un $43,4 \%$ tiene como "input" alguna de estas áreas. A continuación se describe la importancia de las diferentes áreas biotecnológicas por tipo de actividad empresarial.

Dentro de las actividades de las empresas en I $+D$, la salud humana es el área con un mayor número de empresas $(65,8 \%)$. Dentro de la salud humana, el desarrollo de nuevos métodos de diagnóstico ocupa al $46,6 \%$ de las empresas encuestadas y las nuevas terapias al 39,7\%. Dentro de las empresas dedicadas a la salud humana, un $39,6 \%$ se dedican únicamente a nuevos métodos de diagnóstico, un $29,2 \%$ solo a nuevas terapias, y un $31,2 \%$ abordan simultáneamente investigaciones de métodos de diagnóstico y de nuevas terapias.

El segundo campo con mayor actividad en I+D es el de las actividades relacionadas con los procesos industriales, en sus distintas aplicaciones, siendo importante este área para un 32,9\% de las empresas encuestadas. Por subáreas, la obtención de enzimas u otros reactivos fue importante para un $20,5 \%$ del total de empresas encuestadas, alimentos para un $17,8 \%$, los procesos de recuperación de materiales para un $13,7 \%$ y finalmente, actividades industriales para un $11,0 \%$. Entre las empresas con actividad de I+D en procesos industriales, un $45,8 \%$ de las mismas tenía actividad en una sola subárea, un $25,0 \%$ en dos subáreas, un $20,8 \%$ en tres subáreas y un $8,3 \%$ tenía actividad de I+D en las cuatro subáreas definidas dentro de los procesos industriales.

El tercer campo con mayor actividad en I+D es la agricultura, que fue señalado por un $27,4 \%$ de las empresas. En concreto, un $17,8 \%$ de las empresas encuestadas tenían áreas de investigación relacionadas con el mundo de la producción animal, un $12,3 \%$ relacionadas con la producción agraria y un $5,5 \%$ específicamente en acuicultura. La mayoría de las empresas dedicadas a I+D en agricultura se dedican a una sola subárea (75\%), mientras que un $10 \%$ se especializan en dos subáreas y sólo un $5 \%$ de las mismas tiene actividad en las tres grandes subáreas (animales, plantas y acuicultura). La bioinformática, con un $26,0 \%$ del total de empresas encuestadas, tuvo una importancia muy similar a la agricultura. Finalmente, los temas medioambientales ocupan al $15,1 \%$ y las actividades de I+D no específicas se dan en un $19,2 \%$ del total de las empresas encuestadas.

El número de empresas con actividades relacionadas con la producción/comercializción de bienes o servicios es inferior al número de las empresas que tienen actividades en I+D. En concreto, un $74,0 \%$, mientras que todas las empresas encuestadas tenían actividades de I+D. El área relacionada con la sanidad humana sigue siendo la más importante (un 43,8\% respecto del total de empresas y un $59,0 \%$ del total de empresas con producción de bienes y servicios). Un 30,1\% del total de empresas producen nuevos métodos de diagnóstico y un $17,8 \%$ nuevos métodos terápeuticos. La mayoría de las empresas con actividades de producción y comercialización de bienes comercializan o bien métodos de diagnóstico o métodos terapéuticos (un $87,5 \%$ ), y sólo el $12,5 \%$ restante comercializan ambos.

La segunda actividad mayoritaria en producción de bienes y servicios es la de los procesos industriales, realizada por un $23,3 \%$ del total de empresas encuestadas y un $31,5 \%$ de aquéllas con actividades de producción o comercialización de bienes y servicios. Las subáreas más importantes dentro de los procesos industriales fueron alimentos $(12,3 \%$ del total de empresas), enzimas y reactivos $(12,3 \%)$, recuperación de recursos naturales o bioprocesos $(8,2 \%)$ y actividades industriales $(5,5 \%)$. La mayoría de empresas con actividades de producción y comercialización en procesos industriales sólo operaban en una subárea $(58,5 \%)$, mientras que un $23,5 \%$ operaba en dos subáreas, un $17,6 \%$ en tres subáreas y un $5,9 \%$ en las cuatro subáreas anteriormente mencionadas. La agricultura, por otra parte, es importante para un $19,2 \%$ de las empresas encuestadas (un $25,9 \%$ si sólo se consideran las empresas con actividades en bienes y servicios). En este caso, también la subárea de producción animal es la más importante $(13,7 \%$ del total de empresas encuestadas) seguida de cerca por plantas $(11,0 \%)$, con la acuicultura con una importancia mucho menor $(1,4 \%)$. Finalmente, tienen actividad de producción o comercialización en medio ambiente un $11,0 \%$ y un $6,8 \%$ en aplicaciones no específicas del total de empresas encuestadas.

El porcentaje de empresas que utilizan biotecnología como "input" es aún menor, sólo un 43,8\%, comparado con el $74,0 \%$ de las empresas que tenían actividades de producción y comercialización en diferentes campos de la biotecnología y el $100 \%$ de las empresas que tenían actividades de I+D en biotec- 
nología. En este caso las diferencias entre campos de la biotecnología son menores. Tanto la salud humana, como los procesos industriales, la agricultura y la bioinformática tienen la misma importancia, y son utilizadas cada una por un $13,7 \%$ del total de empresas encuestadas (que equivale a un $31,2 \%$ de las empresas que utilizan biotecnologías como "input" en sus actividades). Por otra parte, el medio ambiente y las aplicaciones no específicas son utilizadas como "input" por un $6,8 \%$ cada una (que equivale a un $15,6 \%$ con respecto a las empresas que utilizan biotecnologías como "input" en sus actividades).

Tanto en lo que se refiere a actividades de I+D, o de producción y comercialización de bienes y servicios, existe claramente una especialización muy importante de las empresas biotecnológicas españolas en el sector de la salud humana, y más en el área de métodos de diagnóstico que en el de nuevas terapias. Estos resultados corroboran los resultados de un informe previo de Ernst \& Young sobre biotecnología en Europa, que puso de manifiesto que mientras que la mayoría de las empresas biotecnológicas de países europeos estaban especializadas en el desarrollo de nuevos fármacos, las empresas biotecnológicas españolas estaban especializadas en el desarrollo de nuevos métodos de diagnóstico (Ernst \& Young, 2007). Cabe destacar que el 64\% de los productos comercializados en 2008 por las empresas biotecnológicas españolas estaban relacionados con el área de la salud humana $\mathrm{y}$, en concreto, con los servicios de I + D y los productos de diagnóstico. Una posible explicación a esta especialización es que es consecuencia del Programa Nacional para el mejoramiento de la biotecnología, desarrollado durante los años 1985-1987, donde, entre otras áreas definidas como prioritarias se incluye la investigación en el diagnóstico de las enfermedades más frecuentes (Castro-Martinez y Revilla-Pedreira, 2007).
Financiación pública de la I+D. Las empresas fueron preguntadas por si habían recibido algún tipo de financiación pública para I+D y el origen de la financiación (regional, nacional o europea). El acceso de las empresas a programas públicos de investigación es un aspecto que claramente puede fomentar la colaboración entre empresas y el sector público de I+D (Vega-Jurado y otros, 2009). Un $84,2 \%$ de las empresas afirmaron haber recibido financiación pública, fundamentalmente de programas regionales y nacionales. De hecho, recibieron financiación nacional un $73,1 \%$ y regional un $70,5 \%$ de las empresas encuestadas, por un $32,0 \%$ que recibieron financiación europea. En la mayoría de los casos de empresas que recibieron financiación $(60,9 \%)$, recibieron fondos con dos orígenes (normalmente regional y nacional). Por otra parte, un $12,5 \%$ de las empresas que recibieron fondos sólo los obtuvieron de una fuente (en la mayoría de los casos nacional), y $26,6 \%$ de las empresas recibieron financiación regional, nacional y europea al mismo tiempo.

Con el análisis de la interdependencia y correlación entre los diferentes tipos de financiación y el tamaño de la empresa (ver tabla I), se puede seguir profundizando en el tema de la financiación. En este sentido, se ha podido constatar que existe una correlación positiva al $99 \%$ de confianza (coeficiente de correlación de Spearman $=0,469$ ) entre el número de empresas que han obtenido financiación pública regional y las que han obtenido financiación nacional, lo que significa que es habitual que las empresas obtengan al mismo tiempo financiación regional y nacional. También se ha encontrado una correlación positiva, esta vez significativa al $95 \%$ de confianza (coeficiente de correlación de Spearman $=0,274$ ) entre el tamaño de las empresas y la percepción de fondos con origen europeo. Esto indica que a mayor tamaño

Figura 2. Campos de actividad de las empresas biotecnológicas españolas en sus actividades de $I+D$, producción o comercialización de bienes y servicios, o como "input"

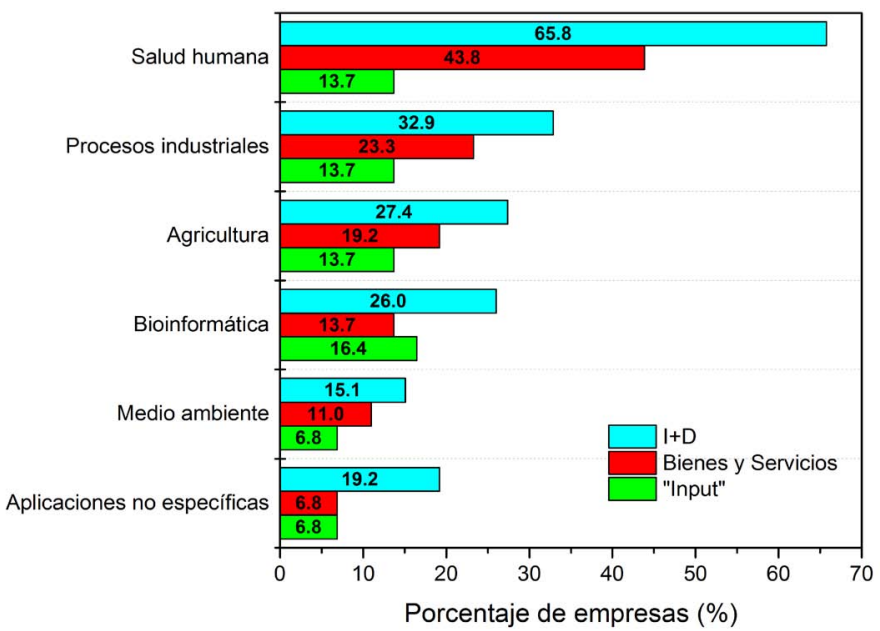


de las empresas, se consigue un acceso más fácil a la financiación europea. Como se ha comentado anteriormente, el acceso a esta fuente de financiación es más restringido que el acceso a financiación regional o nacional, y requiere de un gran esfuerzo de las empresas en la preparación de la propuesta y de búsqueda de socios en otros países. Las empresas más grandes tienen una mayor facilidad para la preparación y desarrollo de proyectos europeos, incluso pueden dedicar parte de su personal a preparar y desarrollar estas propuestas, cosa que es muy difícil en empresas de pequeño tamaño. Obviamente, si tienen capacidad para abordar la participación en proyectos europeos, acceden fácilmente también a la financiación regional y nacional, de hecho, 24 de las 25 empresas que recibieron financiación europea también recibieron financiación regional y nacional. Aunque existen organismos nacionales y regionales, como por ejemplo el Centro para el Desarrollo Tecnológico Industrial (CDTI), o sectoriales (Fundación Genoma España), que tratan de ayudar a participar a las empresas en programas europeos, todavía el acceso a esta fuente de financiación puede mejorarse. Hoy día también está cobrando cada vez más importancia el uso de empresas especializadas en la gestión de la I+D+i que ofrecen entre sus servicios la búsqueda de socios y la preparación de propuestas para ayudar a las pequeñas empresas, pero todavía su uso no está demasiado extendido.

\subsection{Análisis de la colaboración de las empresas} con el sistema público de I+D

Grado de colaboración. El análisis de la colaboración con el sistema público de I+D muestra que el $93,6 \%$ de las empresas ha colaborado en los últimos dos años con algún grupo de investigación del sistema público de I+D. Esto indica que el grado actual de colaboración es muy alto entre las empresas biotecnológicas que se dedican a la $I+D$ y el sistema público de $I+D$, tanto para las empresas completamente dedicadas a la biotecnología como para un número muy importante de las empresas parcialmente dedicadas a la biotecnología. El análisis de dependencia de variables basado en la prueba de Chi-cuadrado de Pearson muestra que las empresas dedicadas al sector salud, en especial las centradas en la búsqueda de nuevas terapias, muestran un mayor grado de colaboración con el sistema público de I+D $\left(X^{2}=4,886 ; p\right.$-valor=0,027). Por tecnología, las empresas centradas en el uso de la bioinformática $\left(X^{2}=5,249 ; p\right.$-valor $\left.=0,022\right)$ y las nanotecnologías $\left(X^{2}=6,915 ; p\right.$-valor $\left.=0,009\right)$ son aquéllas que han mostrado un mayor grado de colaboración con el sistema de I+D

Finalidad de las colaboraciones. Si nos centramos en las empresas que han colaborado previamente con el sistema público de I+D, en la figura 3 se muestra cuál ha sido el propósito de estas colaboraciones. El propósito de esta colaboración ha sido principalmente (en el $83,6 \%$ de los casos), diseñar y llevar a cabo un proyecto de I+D en colaboración entre la empresa y el centro público mediante contrato. En otros casos la empresa ha contratado con el centro público la realización de un proyecto de I+D en dicho centro (49,3\%). En menor medida, la colaboración se ha reducido al suministro de información científica o tecnológica por parte del centro público a la empresa $(21,9 \%)$, y en otros casos, la empresa ha pedido ayuda para la resolución de algún problema puntual de producción (16,4\%). En general, las instituciones implicadas en la realización de proyectos conjuntos de $\mathrm{I}+\mathrm{D}$ con empresas son centros relacionados con

Tabla I. Coeficientes de correlación (Spearman-rho) entre los diferentes tipos de financiación y el tamaño de la empresa

\begin{tabular}{|c|c|c|c|c|c|}
\hline & & $\begin{array}{c}\text { Financiación } \\
\text { Regional }\end{array}$ & $\begin{array}{c}\text { Financiación } \\
\text { Nacional }\end{array}$ & $\begin{array}{c}\text { Financiación } \\
\text { Europea }\end{array}$ & No Empleados \\
\hline \multirow{2}{*}{$\begin{array}{l}\text { Financiación } \\
\text { Regional }\end{array}$} & Coef. Correlación & 1,000 &, $469^{* *}$ &,- 123 &,- 015 \\
\hline & Sig. (2-colas) & . &, 000 & 283 & 899 \\
\hline \multirow{2}{*}{ Financiación Nacional } & Coef. Correlación & $469^{* *}$ & 1,000 &,- 095 & 011 \\
\hline & Sig. (2-tailed) & ,000 & . & 408 & ,926 \\
\hline \multirow{2}{*}{ Financiación Europea } & Coef. Correlación &,- 123 &,- 095 & 1,000 &,$- 274^{*}$ \\
\hline & Sig. (2-tailed) & 283 & 408 & . & 015 \\
\hline \multirow{2}{*}{ No Empleados } & Coef.Correlacion &,- 015 & ,011 & $274^{*}$ & 1,000 \\
\hline & Sig. (2-tailed) & ,899 & ,926 & ,015 & . \\
\hline
\end{tabular}

** Correlación significativa al 99\% de confianza; * Correlación significativa al 95\% de confianza. 
el ámbito de las ciencias de la vida e investigación básica, mientras que las instituciones involucradas en la resolución de problemas puntuales son institutos tecnológicos o centros donde se realiza una investigación más aplicada.

Grado de satisfacción con las colaboraciones. Aunque el grado de colaboración entre las empresas y el sistema público de I+D es muy elevado, es importante conocer también cuál ha sido el grado de satisfacción en esas colaboraciones. En este sentido, la colaboración con el sistema público de investigación fue calificada como muy satisfactoria o bastante satisfactoria por un $70,6 \%$ de las empresas (figura 4). Este grado de satisfacción es relativamente elevado pero indica que casi un $30 \%$ de las empresas no se mostraron satisfechas, en distinto grado, con su colaboración con el sistema público de I+D. En concreto, un 11,5\% de las empresas calificaron la colaboración como poco satisfactoria y un $17,9 \%$ como insatisfactoria o muy insatisfactoria.

Las empresas basadas en conocimiento, como son las empresas biotecnológicas encuestadas, están obligadas a innovar constantemente para lanzar nuevos productos y mantener su posición estratégica (Lerner, 1994; De Rubertis y otros, 2009). Por otro lado, se pone de manifiesto el interés para establecer vínculos con el sistema público de I+D, lo que es estratégicamente ventajoso ya que se reducen los costes de I+D, permiten el acceso a tecnologías más avanzadas, nuevos fondos de financiación pública y aumentan la cartera de patentes, al mismo tiempo que facilitan la creación de vínculos con otras empresas (Stuart y otros, 2007).

Dificultades y obstáculos en la colaboración. Un análisis de las dificultades u obstáculos que se han detectado por parte de las empresas en su colaboración con el sistema público, pone de manifiesto la existencia de problemas que podrían considerarse como estructurales, ya que porcentajes elevados de empresas los consideran como obstáculos importantes, independientemente del grado de satisfacción percibido en la colaboración (figura 5).

Figura 3. Propósito de colaboración entre las empresas biotecnológicas y el sistema público de I+D

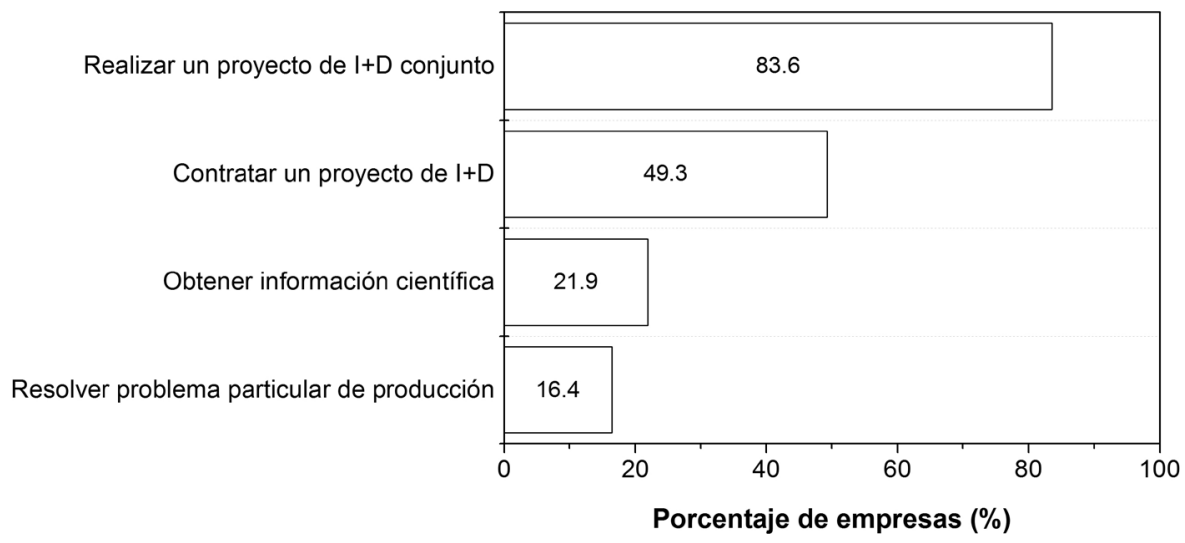

Figura 4. Grado de satisfacción de las empresas con la colaboración con el sistema público de I+D

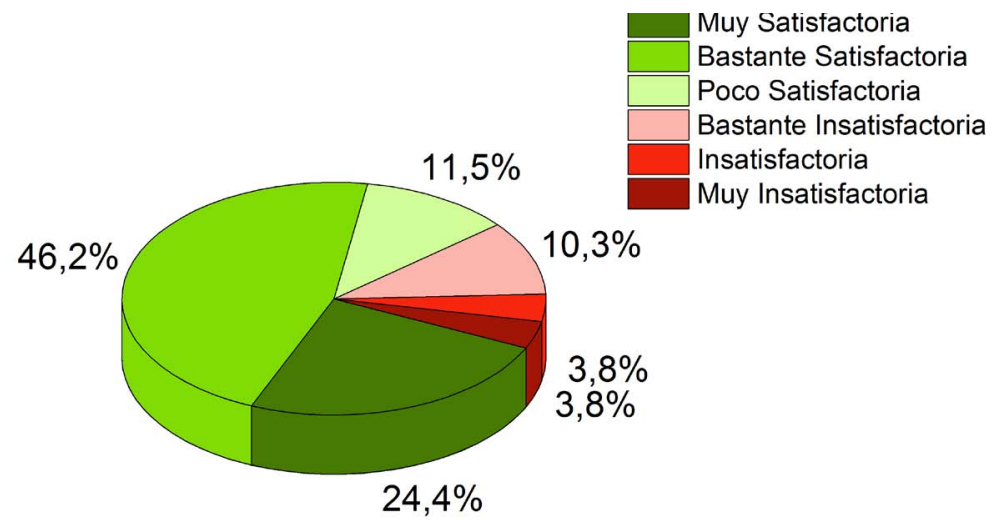


Figura 5. Principales obstáculos encontrados por las compañías en su colaboración con el sistema público de I+D: comparativa entre empresas con percepción positiva o negativa en colaboraciones previas

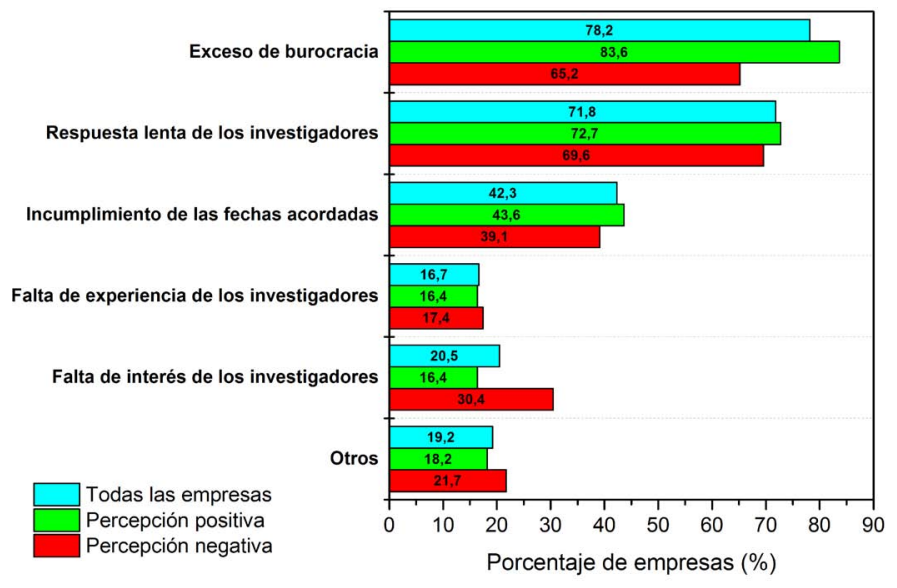

En este apartado de obstáculos aparece en primer lugar el exceso de burocracia, manifestado por el $78,2 \%$ de las empresas, seguido por la lentitud de la respuesta por parte de los investigadores, en opinión del $71,8 \%$ de las empresas. También resulta importante el incumplimiento de los plazos acordados $(42,3 \%)$, lo que está de acuerdo con los otros dos obstáculos previamente comentados. En otro orden de cosas, algunas empresas $(20,5 \%)$ han percibido una falta de interés de los científicos del sistema público de I+D por colaborar con la empresa $y$, finalmente, algunas empresas $(16,7 \%)$ han señalado como obstáculo la falta de experiencia o formación de los investigadores que participaron en los trabajos a realizar.

Otros obstáculos o problemas que han dificultado la colaboración han sido descritos por un 19,2\% de las empresas. Entre estos otros obstáculos citados por las empresas, cabe destacar el papel de las OTTs, que es percibido como burocrático y que plantea discrepancias a la hora de establecer a quién corresponde la propiedad intelectual de los resultados de la colaboración entre la empresa y la institución pública de $I+D$. Este resultado está de acuerdo con el reseñado en el trabajo de Siegel y colaboradores (2003) en el que empresas de distintos emplazamientos de los Estados Unidos consideraban en porcentajes equivalentes un comportamiento por parte de las OTTs de grandes centros universitarios como inflexible y burocrático. Esta excesiva burocracia fue identificada por MoralesGualdrón y otros (2009) como una motivación para la creación de spin-offs por parte de los investigadores. Un $44 \%$ de las 152 spin-offs españolas encuestadas por este autor consideró excesiva la burocracia y las dificultades asociadas a la transferencia de tecnología como las principales razones para la creación de spin-offs. Aparte del papel de las OTTs, otras empresas también consideraron como obstáculo los elevados costes indirectos que gravan el contrato de colaboración, puesto que sumados a los impuestos, incrementan considerablemente el presupuesto de los proyectos. También se destaca en algunos casos la excesiva mentalidad científica o académica de los investigadores, frente a una actitud más enfocada a la tecnología y al mercado por parte de las empresas.

Por otra parte, la figura 5 también recoge la importancia de cada uno de los obstáculos anteriormente comentados, distinguiendo entre las empresas que calificaron su colaboración previa como satisfactoria y aquéllas que la calificaron como insatisfactoria, con el fin de analizar si existen diferencias importantes en los obstáculos nombrados por las empresas dependiendo del grado de satisfacción con colaboraciones previas. Si se tienen en cuenta los tres principales obstáculos encontrados por las compañías en su colaboración con el sistema público de $I+D$, aparecen sólo diferencias importantes en lo que se refiere al exceso de burocracia, mientras que las diferencias son mínimas en aspectos como la lentitud en obtener respuestas a las cuestiones planteadas o en lo que concierne al incumplimiento de los plazos acordados para la finalización de la colaboración por parte de los investigadores del sistema público. Si el exceso de burocracia fue el obstáculo señalado por un mayor número de empresas (un $78,2 \%$ ), este obstáculo es de mucha mayor importancia para las empresas satisfechas (un $83,6 \%$ de las mismas) y de menor importancia para las empresas insatisfechas (un $65,2 \%$ de las mismas). De hecho, para las empresas poco satisfechas con las colaboraciones previas, la respuesta lenta de los investigadores supera al obstáculo del exceso de burocracia como la principal barrera en su colaboración $(69,6 \%$ frente al $65,2 \%$ de las empresas insatisfechas). Parece que el motivo de la insatisfacción en la colaboración con el sistema público de I+D tiene varias causas, no sólo un exceso de burocracia, que suele 
ser conocido ya por las empresas y, por tanto, en mayor o menor medida asumido cuando se pretende realizar algún tipo de colaboración con el sistema público de I+D. El exceso de burocracia en sí no parece ser suficientemente importante como para determinar el grado de satisfacción final en la colaboración con el sistema público de I+D.

En otros aspectos considerados como barreras, las opiniones manifestadas por las empresas satisfechas y las insatisfechas son parecidas (falta de experiencia de los investigadores y otras barreras), sin embargo, existe una diferencia importante en cuanto a una percepción que se podría calificar de subjetiva y que se refiere al interés de los científicos por la colaboración propuesta por las empresas. Si la falta de interés de los científicos por la colaboración fue señalada por un $20,5 \%$ de las empresas, este porcentaje es prácticamente el doble en el caso de las empresas insatisfechas $(30,4 \%$ de las mismas) que en las empresas satisfechas $(16,4 \%$ de las mismas). Lo que podría indicar que es un aspecto que no toleran las empresas y que puede que sea una de las per- cepciones que pueden determinar de forma más importante el grado de satisfacción global de la empresa con el sistema público de I+D.

La tabla II, que analiza los coeficientes de correlación entre los obstáculos encontrados por las empresas y el grado de satisfacción, confirma estas observaciones. Mientras que el exceso de burocracia es uno de los factores de menor importancia sobre el grado de satisfacción, el factor más significativo que determina el grado de satisfacción de las empresas es la falta de interés de los investigadores, significativo al $99 \%$ de confianza y con un coeficiente de correlación de $-0,461$. Aparte, la tabla II puede constatar otros aspectos como son la interrelación entre obstáculos. Como puede observarse, la lentitud de la respuesta está lógicamente correlacionada al $99 \%$ de confianza con el fallo en el cumplimiento de los plazos acordados (coeficiente de correlación 0,483 ) y el exceso de burocracia está interrelacionada al $95 \%$ de confianza con la lentitud en la respuesta (coeficiente de correlación 0,257).

Tabla II. Coeficientes de correlación entre los obstáculos encontrados por las empresas en su colaboración con el sistema público de I+D y grado de satisfacción con la colaboración

\begin{tabular}{|c|c|c|c|c|c|c|c|}
\hline & & $\begin{array}{c}\text { Falta de } \\
\text { interés de los } \\
\text { investigadores }\end{array}$ & $\begin{array}{c}\text { Falta de } \\
\text { experiencia } \\
\text { de los } \\
\text { investigadores }\end{array}$ & $\begin{array}{l}\text { Lentitud en } \\
\text { la respuesta }\end{array}$ & $\begin{array}{c}\text { Fallo en el } \\
\text { cumplimiento } \\
\text { de los plazos } \\
\text { acordados }\end{array}$ & $\begin{array}{c}\text { Excesiva } \\
\text { burocracia }\end{array}$ & $\begin{array}{c}\text { Grado de } \\
\text { satisfacción } \\
\text { con la } \\
\text { colaboración }\end{array}$ \\
\hline \multirow{2}{*}{$\begin{array}{l}\text { Falta de } \\
\text { interés de los } \\
\text { investigadores }\end{array}$} & $\begin{array}{l}\text { Coef. } \\
\text { Correlación }\end{array}$ & 1,000 &,- 005 & 111 & ,020 &,- 035 &,$- 461^{* *}$ \\
\hline & $\begin{array}{l}\text { Sig. } \\
\text { (2-tailed) }\end{array}$ & . & ,967 & ,333 & ,862 & 763 & ,000 \\
\hline \multirow{2}{*}{$\begin{array}{l}\text { Falta de } \\
\text { experiencia } \\
\text { de los } \\
\text { investigadores }\end{array}$} & $\begin{array}{l}\text { Coef. } \\
\text { Correlación }\end{array}$ &,- 005 & 1,000 & 111 & ,020 & 129 &,- 043 \\
\hline & $\begin{array}{l}\text { Sig. } \\
\text { (2-tailed) }\end{array}$ & ,967 & . & ,333 & ,862 & 259 & 710 \\
\hline \multirow{2}{*}{$\begin{array}{l}\text { Lentitud en la } \\
\text { respuesta }\end{array}$} & $\begin{array}{l}\text { Coef. } \\
\text { Correlación }\end{array}$ & 111 & 111 & 1,000 &, $483^{* *}$ &, $257^{*}$ &,- 138 \\
\hline & $\begin{array}{l}\text { Sig. } \\
\text { (2-tailed) }\end{array}$ & ,333 & ,333 & . & ,000 & ,023 & ,228 \\
\hline \multirow{2}{*}{$\begin{array}{l}\text { Fallo en el } \\
\text { cumplimiento } \\
\text { de los plazos } \\
\text { acordados }\end{array}$} & $\begin{array}{l}\text { Coef. } \\
\text { Correlación }\end{array}$ & ,020 & ,020 &, $483^{* *}$ & 1,000 &, 144 &,- 173 \\
\hline & $\begin{array}{l}\text { Sig. } \\
\text { (2-tailed) }\end{array}$ & ,862 & ,862 & ,000 & . & 208 & 131 \\
\hline \multirow{2}{*}{$\begin{array}{l}\text { Excesiva } \\
\text { burocracia }\end{array}$} & $\begin{array}{l}\text { Coef. } \\
\text { Correlación }\end{array}$ &,- 035 & 129 & $257^{*}$ & 144 & 1,000 &,- 130 \\
\hline & $\begin{array}{l}\text { Sig. } \\
\text { (2-tailed) }\end{array}$ & ,763 & 259 & ,023 & 208 & . & ,256 \\
\hline \multirow{2}{*}{$\begin{array}{l}\text { Clasificación de } \\
\text { la colaboración }\end{array}$} & $\begin{array}{l}\text { Coef. } \\
\text { Correlación }\end{array}$ &,$- 461^{* *}$ &,- 043 &,- 138 &,- 173 &,- 130 & 1,000 \\
\hline & $\begin{array}{l}\text { Sig. } \\
\text { (2-tailed) }\end{array}$ & ,000 & ,710 & ,228 & ,131 & ,256 & . \\
\hline
\end{tabular}

** Correlación significativa al 99\% de confianza, * Correlación significativa al 95\% de confianza. 
Por otra parte, estos obstáculos son percibidos de forma diferente dependiendo del tipo de biotecnología utilizada por la empresa (tabla III). Las empresas relacionadas con procesos biotecnológicos consideran la falta de interés de los científicos en algún área concreta como el principal obstáculo encontrado en la colaboración, con un valor de Chi-cuadrado de Pearson $\left(X^{2}\right)$ de 4,453 . Por otro lado, los valores de $X^{2}$ muestran que estas empresas han encontrado diferencias entre la lentitud de la respuesta $\left(X^{2}=5,293\right)$ y el fallo en el cumplimiento de los plazos acordados $\left(X^{2}=4,355\right)$. Las empresas que utilizan biotecnologías basadas en DNA y proteínas también han identificado el incumplimiento de los plazos acordados o la lentitud en la respuesta por parte de los investigadores del sistema público de I+D como los obstáculos más importantes en la colaboración. Estas empresas se dedican principalmente al sector de la salud humana, donde es necesario generar productos rápidamente para poder competir en mercados tan competitivos como el farmacéutico. El exceso de burocracia por parte de la administración pública puede crear dificultades en el normal desarrollo de un proyecto, sobre todo en el cumplimento de los plazos acordados, ya que entre otros problemas, puede provocar el retraso de la contratación del personal necesario para llevar a cabo el proyecto o no tener disponibles los materiales y equipos necesarios para realizar la investigación y por tanto, demorando la obtención de resultados.
También se han detectado diferencias en la percepción de los obstáculos según el área de aplicación de la empresa. La falta de interés de los investigadores ha sido percibida como el principal obstáculo por las empresas especializadas en diagnóstico con un valor de $x^{2}$ de 9,542 ( $p$-valor $=0,009)$. Este obstáculo es también muy importante para las empresas dedicadas a acuicultura $\left(X^{2}=9,290 ; p\right.$-valor $\left.=0,002\right)$ y la producción animal $\left(X^{2}=5,886 ; p\right.$-valor $\left.=0,015\right)$. Las empresas cuyo sector de aplicación es la agricultura, en concreto lo relacionado con alimentación animal, han encontrado como principal obstáculo la lentitud en la respuesta $\left(X^{2}=4,105 ; p\right.$-valor $\left.=0,043\right)$. Por otro lado, las empresas dedicadas a los procesos industriales encuentran como obstáculo principal la falta de cualificación o experiencia de los investigadores $\left(X^{2}=4,735 ; p\right.$-valor $\left.=0,030\right)$.

Futuras colaboraciones. Finalmente, las empresas fueron preguntadas si planeaban colaborar en un futuro próximo con el sistema público de I+D. De las 48 empresas que contestaron a la pregunta (un $61,6 \%$ del total de empresas encuestadas), un $81,2 \%$ afirmó que volvería a colaborar con el sistema público de I+D en un futuro frente a un $18,8 \%$ que no planean colaborar.

Si se analiza la respuesta teniendo en cuenta qué empresas habían calificado como satisfactorias o insatisfactorias sus colaboraciones previas, los resultados son también muy significativos.

Tabla III. Valores de Chi-cuadrado de Pearson para los obstáculos encontrados por las empresas, según la tecnología utilizada, en su colaboración con el sistema público de I+D

\begin{tabular}{|c|c|c|c|c|c|}
\hline & $\begin{array}{l}\text { Fallo en el } \\
\text { cumplimiento } \\
\text { de los plazos } \\
\text { acordados }\end{array}$ & $\begin{array}{l}\text { Lentitud } \\
\text { en la } \\
\text { respuesta }\end{array}$ & $\begin{array}{c}\text { Falta de } \\
\text { experiencia de los } \\
\text { investigadores }\end{array}$ & $\begin{array}{l}\text { Exceso de } \\
\text { burocracia }\end{array}$ & $\begin{array}{c}\text { Falta de } \\
\text { interés de los } \\
\text { investigadores }\end{array}$ \\
\hline DNA & $\begin{array}{c}8,247 \\
(0,004)\end{array}$ & $\begin{array}{c}4,237 \\
(0,040)\end{array}$ & $\begin{array}{c}1,709 \\
(0,161)\end{array}$ & $\begin{array}{c}0,815 \\
(0,367)\end{array}$ & $\begin{array}{c}0,827 \\
(0,363)\end{array}$ \\
\hline $\begin{array}{l}\text { Proteinas y otras } \\
\text { Macromoléculas }\end{array}$ & $\begin{array}{c}6,019 \\
(0,014)\end{array}$ & $\begin{array}{c}7,389 \\
(0,007)\end{array}$ & $\begin{array}{c}1,130 \\
(0,288)\end{array}$ & $\begin{array}{c}0,482 \\
(0,488)\end{array}$ & $\begin{array}{c}0,126 \\
(0,723)\end{array}$ \\
\hline $\begin{array}{c}\text { Procesos } \\
\text { Biotecnológicos }\end{array}$ & $\begin{array}{c}4,355 \\
(0,037)\end{array}$ & $\begin{array}{c}5,293 \\
(0,021)\end{array}$ & $\begin{array}{c}1,954 \\
(0,162)\end{array}$ & $\begin{array}{c}0,790 \\
(0,374)\end{array}$ & $\begin{array}{c}4,453 \\
(0,035)\end{array}$ \\
\hline $\begin{array}{l}\text { Cultivo e Ingeniería } \\
\text { de Células/Tejidos }\end{array}$ & $\begin{array}{c}0,177 \\
(0,674)\end{array}$ & $\begin{array}{c}1,990 \\
(0,158)\end{array}$ & $\begin{array}{c}0,336 \\
(0,562)\end{array}$ & $\begin{array}{c}0,237 \\
(0,601)\end{array}$ & $\begin{array}{c}0,732 \\
(0,392)\end{array}$ \\
\hline Bioinformatica & $\begin{array}{c}3,311 \\
(0,191)\end{array}$ & $\begin{array}{c}2,027 \\
(0,363)\end{array}$ & $\begin{array}{c}0,626 \\
(0,731)\end{array}$ & $\begin{array}{c}3,393 \\
(0,183)\end{array}$ & $\begin{array}{c}0,136 \\
(0,934)\end{array}$ \\
\hline Nanobiotecnología & $\begin{array}{c}0,724 \\
(0,395)\end{array}$ & $\begin{array}{l}1,298 \\
(0,255)\end{array}$ & $\begin{array}{c}0,212 \\
(0,645)\end{array}$ & $\begin{array}{c}0,493 \\
(0,483)\end{array}$ & $\begin{array}{c}0,114 \\
(0,735)\end{array}$ \\
\hline $\begin{array}{c}\text { Genes y Vectores de } \\
\text { RNA }\end{array}$ & $\begin{array}{l}1,548 \\
(0,213)\end{array}$ & $\begin{array}{c}2,007 \\
(0,157)\end{array}$ & $\begin{array}{c}0,008 \\
(0,928)\end{array}$ & $\begin{array}{c}2,763 \\
(0,096)\end{array}$ & $\begin{array}{l}1,583 \\
(0,208)\end{array}$ \\
\hline
\end{tabular}


Si un 55\% de las empresas con experiencia satisfactoria pensaban realizar algún tipo de colaboración en el futuro próximo, un $5 \%$ contestó que no y un $29 \%$ no sabe/no contesta; en el caso de las empresas con percepción negativa, sorprendentemente, todavía un $42 \%$ de las mismas planeaba alguna colaboración en el futuro cercano, mientras que un $29 \%$ respondía negativamente y un $29 \%$ no sabe/no contesta. Está claro que la experiencia insatisfactoria en colaboraciones previas marca a las empresas, pero todavía un porcentaje importante de empresas planean colaborar en un futuro próximo con el sistema público de I+D a pesar de las dificultades encontradas.

\section{CONCLUSIONES}

Las empresas biotecnológicas españolas con actividades de I+D son empresas de pequeño tamaño, altamente especializadas en el sector de la salud humana, sobre todo en la investigación de nuevos métodos diagnósticos, y en menor medida, nuevas terapias. También son importantes las áreas de procesos industriales y agricultura, y ya en menor medida, la bioinformática, el medio ambiente y otras aplicaciones no específicas. De las empresas que realizan actividades de $\mathrm{I}+\mathrm{D}$, un $74,0 \%$ también realizan actividades de producción o comercialización de bienes y servicios y un $43,4 \%$ utilizaron la biotecnología como "input" para su actividad. Un $84,2 \%$ de las empresas con actividades en I+D recibió algún tipo de financiación pública para desarrollar sus actividades de I+D, sobre todo nacional y regional, y en menor medida, europea, aunque normalmente esta última fuente de financiación está restringida a las empresas de mayor tamaño.

La colaboración de las empresas con el sistema público de I+D es ya muy alta, pues un $93,6 \%$ de las empresas encuestadas tuvieron algún tipo de colaboración con el sistema público de I+D en los últimos dos años. En este sentido, parece importante el hecho de haber recibido financiación pública para establecer más colaboraciones con el sistema público de I+D. La mayoría de las colaboraciones son para realizar un proyecto de $\mathrm{I}+\mathrm{D}$ conjunto $(83,6 \%$ de las colaboraciones) o para contratar un proyecto de I+D que se hará en el centro público de I+D (49,3\%). En menor medida, la colaboración ha consistido en la obtención de información científica $(21,9 \%)$ o para resolver un problema particular de producción $(16,4 \%)$

No sólo el grado de colaboración entre las empresas y el sistema público de $\mathrm{I}+\mathrm{D}$ en el sector biotecnológico es alto, sino que el grado de satisfacción de las empresas con las colaboraciones previas con el sistema público de I+D es muy alto. Un $70,6 \%$ de las empresas que colaboraron con el sistema público de I+D consideraron como bastante satisfactoria o muy satisfactoria la colaboración. Sin embargo, todavía un $11,5 \%$ de las empresas la calificaron como poco satisfactoria y un $17,9 \%$ como insatisfactoria o muy insatisfactoria.
Cuando se analizaron los principales obstáculos o barreras encontrados por las empresas, el exceso de burocracia (por un $78,2 \%$ de los encuestados) y la respuesta lenta de los investigadores (por un $71,8 \%$ de los encuestados) fueron los principales obstáculos señalados. En menor medida, fueron señalados el incumplimiento de fechas acordadas $(42,3 \%)$, la falta de experiencia de los investigadores $(16,7 \%)$, la falta de interés de los investigadores $(20,5 \%)$ y otros $(19,2 \%)$, fundamentalmente relacionados con el papel de las OTTs. No obstante, se ha podido demostrar que la excesiva burocracia, aunque señalada por la mayoría de los encuestados, no determina el grado de satisfacción global con la colaboración. Sin embargo, la falta de interés de los investigadores fue el obstáculo que más determinó el grado de satisfacción. En cualquier caso, tanto las empresas con colaboraciones previas consideradas como satisfactorias como también en un importante porcentaje en las empresas con colaboraciones previas considerados como insatisfactorias, planean establecer más colaboraciones con el sistema público de $\mathrm{I}+\mathrm{D}$ en un futuro próximo.

Algunas empresas consideran que los científicos no han mostrado interés por el problema objeto del estudio planteado, o que su solución se ha dejado en manos de personal no cualificado suficientemente. Esta falta de interés de los científicos en la investigación propuesta por las empresas puede estar relacionada, entre otros aspectos, a las cláusulas de confidencialidad incluidas en los proyectos de colaboración, lo que dificulta la difusión y publicación de los resultados que se obtendrán, debido a las prácticas de evaluación de la investigación, que se basa en la medida del esfuerzo en la publicación de artículos científicos (ManjarrésHenríquez y otros, 2008). Estos problemas pueden estar vinculados a un exceso de "mentalidad académica", en oposición a una "mentalidad de negocios", como también se ha detectado en otros estudios (Siegel y otros, 2003). En este sentido, también se puede señalar como un factor determinante la falta de incentivos a los científicos de los centros públicos de investigación para mejorar la colaboración con centros de investigación de las empresas públicas (Morales-Gualdrón y otros, 2009). Aquellas instituciones públicas, que por razones políticas o económicas, han fomentado la colaboración con la industria tienen un importante número de investigadores que participan en proyectos de colaboración (Belkhodja y Landry, 2007). Sin embargo, también se ha observado que si la financiación privada supera el $75 \%$ de la financiación total de la institución tiene un efecto negativo en la productividad científica.

En general, las políticas del gobierno dirigidas a fomentar la transferencia de conocimiento y la colaboración científica entre el sector público de I+D y el sector privado están dirigidas fundamentalmente a incrementar la colaboración de las empresas con los OPIs, como es el caso del sector de 
la biotecnología, pero por lo general no incluyen claros incentivos para el sector público.

Los resultados obtenidos sugieren la conveniencia de establecer políticas y programas públicos para fomentar la transferencia de conocimiento y tecnología desde los centros públicos de investigación a las empresas. Estas políticas y estos programas deberían incluir, por un lado, los mecanismos más flexibles de las OTTs sobre los diferentes aspectos aquí considerados, incluyendo los derechos de propiedad intelectual, que participan en la formulación del contrato. Por otro lado, parece conveniente mejorar la información a las empresas sobre aspectos económicos implicados en la formulación de los contratos con los organismos públicos de investigación. Esto puede ser importante, al menos para algunas de las empresas encuestadas que tienen dificultades para aceptar que el coste total del contrato de investigación tiene que incluir los gastos generales y el IVA. Con el fin de lograr una relación beneficiosa entre empresas e investigadores, la participación de los científicos del sistema público de investigación debe ser incentivado para una colaboración más eficiente, a través de reglamentos valorar positivamente su participación, tanto económica como académicamente.

\section{BIBLIOGRAFÍA}

Abramo, G.; DAngelo, C.A.; Di Costa, F.; Solazzi, M. (2009), University-industry collaboration in Italy: A bibliometric examination. Technovation, vol. 29, 498-507. http://dx.doi.org/10.1016/j. technovation.2008.11.003

Albert, A.; Granadino, B.; Plaza, L.M. (2007). Scientific and Technological Performance Evaluation of the Spanish Council for Scientific Research (CSIC) in the field of biotechnology. Scientometrics, vol. 70(1), 41-51. http://dx.doi. org/10.1007/s11192-007-0103-y

Albert, A.; Plaza, L.M. (2004). Análisis de la producción científica española citada en patentes biotecnológicas en EE.UU. Revista Española de Documentación Científica, vol. 27 (2), 212-220.

Al-Laham, A.; Tzabbar D.; Amburgey T.L. (2011). The dynamics of knowledge stocks and knowledge flows: innovation consequences of recruitment and collaboration in biotech. Industrial and Corporate Change, vol. 20(2), 555583. http://dx.doi.org/10.1093/icc/dtr001

Almeida, P.; Hohberger, J.; Parada, P. (2011). Individual scientific collaborations and firm-level innovation. Industrial and Corporate Change, vol. 20(6), 1571-1599. http://dx.doi.org/10.1093/ icc/dtr030

Asociación Española de Bioempresas (ASEBIO) (2011). Informe ASEBIO 2010. Madrid: Asociación Española de Bioempresas.

Asociación Española de Bioempresas (ASEBIO) (2012). Informe ASEBIO 2011. Madrid: Asociación Española de Bioempresas.
Asociación Española de Bioempresas (ASEBIO) (2013). Informe ASEBIO 2012. Madrid: Asociación Española de Bioempresas.

Belkodja, O.; Landry, R. (2007). The Triple-Helix collaboration: Why do researchers collaborate with industry and the government?. What are the factors that influence the perceived barriers? Scientometrics, vol. 70(2), 301-302. http:// dx.doi.org/10.1007/s11192-007-0205-6

Cardozo, R.; Ardichvili, A.; Strauss, A. (2011). Effectiveness of university technology transfer: an organizational population ecology view of a maturing supplier industry. Journal of Technology Transfer, vol. 36, 173-202. http://dx.doi. org/10.1007/s10961-010-9151-1

Castro-Martínez, E.; Revilla-Pedreira, R. (2007). La participación del CDTI en los inicios de las políticas de fomento de la I+D sobre biotecnología en España. En: Tiempos de ciencia y de política: homenaje a Emilio Muñoz. Madrid: Ed. CSIC.

De Rubertis, F.; Fleck, R.; Lanthaler, W. (2009). Six secrets to success- How to build a sustainable Biotech business. Nature, vol. 27(7), 595-597.

Ernst and Young (2007). Beyond Borders. Global Biotechnology Report 2007. London; Ernst and Young International.

Etzkowitz, H.; Leydesdorff, L. (2000). The dynamics of innovation: from National Systems and Mode 2 to a Triple Helix of university-industrygovernment relations. Research Policy, vol. 29, 109-123. http://dx.doi.org/10.1016/S00487333(99)00055-4

Fundación Genoma España (2011). Relevancia Empresarial y Financiera. En: Relevancia de la biotecnología en España 2011. Fundación para el Desarrollo de la Investigación en Genómica y Proteómica Genoma España, Madrid, España.

George, G.; Zahra, SA.; Wood, D.R. (2002). The Effects of Business-University Alliances on Innovative Output and Financial Performance: a Study of Publicly Traded Biotechnology Companies. Journal of Business Venturing, Vol. 17(6), 577-609. http://dx.doi.org/10.1016/S08839026(01)00069-6

Hayton, J.C.; Sehili, S.; Scarpello, V. (2010). Why do firms join consortial research centers?. An empirical examination of firms, industry and environmental antecedents. Journal of Technology Transfer, vol. 35, 494-510. http://dx.doi. org/10.1007/s10961-010-9157-8

Katz, J.S.; Martin, B.R. (1997). What is research collaboration? Research Policy, vol. 26, 1-18. http://dx.doi.org/10.1016/S00487333(96)00917-1

Lerner, J. (1994). The Importance of Patent Scope - an Empirical-Analysis. RAND Journal of Economics, vol. 25(2), 319-33. http://dx.doi. org/10.2307/2555833

Manjarrés-Henriquez, L.; Gutiérrez-Gracia, A.; Vega-Jurado, J. (2008). Coexistence of university-industry relations and academic research: 
Barrier to or incentive for scientific productivity. Scientometrics, vol. 73 (3), 561-576. http:// dx.doi.org/10.1007/s11192-007-1877-7

Martinelli, A.; Meyer, M.; von Tunzelmann, N. (2008). Becoming an entrepreneurial university? A case study of knowledge exchange relationships and faculty attitudes in a mediumsized, research-oriented university. Journal of Technology Transfer, vol. 33, 259-283. http:// dx.doi.org/10.1007/s10961-007-9031-5

Morales-Gualdrón, S.T.; Gutiérrez-Gracia, A.; Roig Dobón, S. (2009). The entrepreneurial motivation in academia: a multidimensional construct. International. Entrepreneurship and Management Journal, vol. 5, 301-317. http://dx.doi. org/10.1007/s11365-009-0113-8

Muscio, A. (2010). What drives the university use of technology transfer offices? Evidences from Italy. Journal of Technology Transfer, vol. 35, 110-202. http://dx.doi.org/10.1007/s10961009-9121-7

OCDE (2005a). A Framework for Biotechnology Statistics. Paris, Organisation for Economic CoOperation and Development. En: http://www. oecd.org/sti/sci-tech/34935605.pdf [consultado: 7 de mayo de 2010].

OCDE (2005b). Statistical definition of Biotechnology. En: http://www.oecd.org/science/inno/ statisticaldefinitionofbiotechnology.htm [consultado: 7 de mayo de 2010].

OCDE (2012). Key Biotechnology Indicators 2012. En: http://www.oecd.org/sti/inno/keybiotechnologyindicators.htm [consultado: 21 de febrero de 2013].

Oliver, A.L. (2004). Biotechnology entrepreneurial scientists and their collaborations. $R e-$ search Policy, vol. 33, 583-597. http://dx.doi. org/10.1016/j.respol.2004.01.010

Ramos-Vielba, I.; Fernández-Esquinas, M.; Espinosa de los Monteros, E. (2009). Measuring university-industry collaboration in a regional innovations system. Scientometrics, vol. 84(3), 649-667. http://dx.doi.org/10.1007/s11192009-0113-z

Santoro, M.D.; Gopalakrishnan, S. (2000). The Institutionalization of Knowledge Transfer Activities Within Industry-University Collaborative
Ventures. Journal of Engineering and Technology Management, vol. 17(3-4), 299-319. http:// dx.doi.org/10.1016/S0923-4748(00)00027-8

Schartinger, D.; Rammer, C.; Fischer, M.M.; Frohlich, J. (2002). Knowledge Interactions between Universities and Industry in Austria: Sectorial Patterns and Determinants. Research Policy, vol. 31(3), 303-28. http://dx.doi.org/10.1016/ S0048-7333(01)00111-1

Siegel, D.S.; Wessner, C.; Binks, M.; Lockett, A. (2003). Policies Promoting Innovation in Small Firms: Evidence From the US and UK. Small Business Economics, vol. 20(2), 121-127. http:// dx.doi.org/10.1023/A:1022271914246

Stuart, T.E.; Ozdemir, S.Z.; Ding, W.W. (2007). Vertical Alliance Networks: the Case of University-Biotechnology-Pharmaceutical Alliance Chains. Research Policy, vol. 36(4), 477-498. http://dx.doi.org/10.1016/j.respol.2007.02.016

Thursby, J.; Thursby, M. (2011). University-industry linkages in nanotechnology and biotechnology: evidence on collaborative patterns for new methods of inventing. Journal of Technology Transfer, vol. 36, 605-623. http://dx.doi. org/10.1007/s10961-011-9213-z

Vega-Jurado, J.; Gutiérrez-Gracia, A.; Fernándezde-Lucio, I. (2009). Does external knowledge sourcing matter for innovation? Evidence from the Spanish manufacturing industry. Industrial and Corporate Change, vol. 18(4), 637-670. http://dx.doi.org/10.1093/icc/dtp023

Yong, S. L. (2000). The sustainability of university-industry research collaboration: An empirical Assessment. Journal of Technology Transfer, vol. 25(2), 111-133. http://dx.doi. org/10.1023/A:1007895322042

Zaheer, A.; George, V.P. (2004). Reach out or reach within? Performance implications of alliances and location in biotechnology. Managerial and Decision Economics, vol. 25(6-7), 437-452. http://dx.doi.org/10.1002/mde.1200

Zhang, J.; Baden-Fuller, C.; Mangematin, V. (2007). Technological knowledge base, R\&D organization structure and alliance formation: Evidence from the biopharmaceutical industry. Research Policy, vol. 36, 515-528. http://dx.doi. org/10.1016/j.respol.2007.02.015 